\title{
Perineural spread of malignant melanoma from the mandible to the brachial plexus: case report
}

\author{
Carlos E. Restrepo, MD, ${ }^{1}$ Robert J. Spinner, MD, ${ }^{1}$ B. Matthew Howe, MD, ${ }^{2}$ Mark E. Jentoft, MD, ${ }^{3}$ \\ Svetomir N. Markovic, MD, PhD, ${ }^{4}$ and Daniel H. Lachance, MD ${ }^{5}$ \\ Departments of ${ }^{1}$ Neurosurgery, ${ }^{2}$ Radiology, ${ }^{3}$ Laboratory Medicine and Pathology, ${ }^{4}$ Oncology, and ${ }^{5}$ Neurology, Mayo Clinic, \\ Rochester, Minnesota
}

\begin{abstract}
Perineural spread is a well-known mechanism of dissemination of head and neck malignancies. There are few reports of melanoma involving the brachial plexus in the literature. To their knowledge, the authors report the first known case of perineural spread of malignant melanoma to the brachial plexus. Clinicoradiological and anatomopathological correlation is presented, highlighting the importance of peripheral nerve communications in perineural spread.
\end{abstract}

http://thejns.org/doi/abs/10.3171/2014.12.JNS14852

KEY WORDS perineural spread; malignant melanoma; cervical plexus; brachial plexus; peripheral nerve; oncology

$\mathrm{P}$ ERINEURAL spread is increasingly recognized as a relatively common mechanism of metastasis in many types of malignancies, particularly those involving the head and neck region. This mechanism of tumor spread for metastatic melanoma is well known to occur along the cranial nerves and cervical plexus..$^{4,9,14,16-18} \mathrm{We}$ describe a rare example of documented perineural spread of melanoma, arising in the mandible and extending along a cranial nerve, the cervical plexus, and into the brachial plexus. We correlate the clinicoradiological features and propose an anatomopathological explanation to support the mechanism of perineural spread in this case.

\section{Case Report}

History

A 48-year-old right-handed woman presented in 2010 with a several-month history of progressive numbness, tingling, itching, and atrophy affecting the skin and muscles of the right neck. MRI of the cervical spine revealed right C2-3 and C3-4 foraminal masses (Fig. 1 left). The patient underwent observation. Repeat MRI performed 7 months later showed spinal cord displacement at the level of C-2 (Fig. 1 right). The patient underwent an open biopsy-the findings of which were consistent with a neurofibromaand 1 week later, C2-4 laminectomy, decompression, and fusion. The lesion was partially resected. Postoperatively, the patient received 45-Gy radiotherapy to a field from the top of $\mathrm{C}-2$ to the bottom of $\mathrm{C}-6$, including the spinal cord as well as an 18-Gy boost to the right-sided upper cervical roots and nerves. Brain MRI findings at that time were normal. The brachial plexus was not imaged.

Over the next 2 years the patient had persistent cervical discomfort and progressive pain in her right shoulder and then the biceps region. Neither partial removal of the instrumentation nor steroid injections provided relief of her pain, which progressed as time passed. In 2013 she was evaluated at a tertiary care facility when she noted weakness in shoulder abduction and external rotation. Imaging now revealed plexiform lesions involving multiple cervical nerves and the upper brachial plexus. Pathological specimens were reviewed and the findings were interpreted as hybrid schwannoma/neurofibroma. The patient was thought to have a segmental form of schwannomatosis.

The patient had a history of melanoma. In 2001, she had a lesion excised from her trunk with no local recurrence. In 2004, she had a nearly 2-cm lesion excised from the angle of the right jaw, a fusion of lentigo maligna and desmoplastic types. It was invasive to Clark's Level IV and Breslow's ${ }^{3}$ depth $3 \mathrm{~mm}$.The mitotic rate was 0 . Local perineural invasion was evident in the specimen. Tumor was present at the biopsy margins. The $B R A F$ was wild type. Findings from the sentinel cervical lymph node biopsy were negative.

\section{Physical Examination}

In February 2014, the patient presented to our institution. Physical examination revealed atrophic right neck 

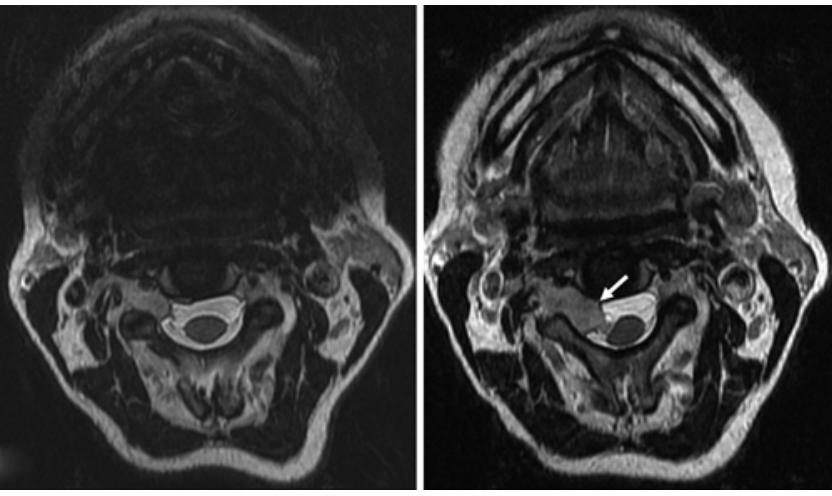

FIG. 1. Axial T2-weighted MR images through the level of the C-2 foramina. Left: Initial image of the cervical spine demonstrating the softtissue mass in the right C-2 neuroforamen. Right: Image through the level of C-2 obtained 7 months later. Note the enlargement of the C-2 mass, which now protrudes into the central canal with some mass effect on the cervical cord (arrow). muscles, including the sternocleidomastoid. The patient had a palpable painful mass in the neck, which corresponded to the course of the spinal accessory nerve. There was no cervical lymphadenopathy. Abduction was limited to $30^{\circ}$. The patient had Medical Research Council Grade 2 external rotation and Grade 4 elbow flexion (both biceps and brachioradialis); elbow extension was Grade 4. The patient had no trapezius activation, but other cranial nerve functions were normal. There was decreased sensation in the right neck, ear, chest, and proximal arm. Biceps and brachioradialis muscle reflexes in the right arm were absent. Percussion of the supraclavicular region produced radiated paresthesias in the shoulder and elbow regions.

\section{Additional Testing}

The patient refused an electromyography/nerve conduction study. Chest radiography showed an elevated right-sided hemidiaphragm. T2-weighted and gadolinium-enhanced MRI of the head, neck, and brachial plexus demonstrated extensive abnormality (Fig. 2A). The great auricular nerve and lesser occipital nerve appeared abnormal, extending from C-2 to C-4 laterally (Fig. 2B). The
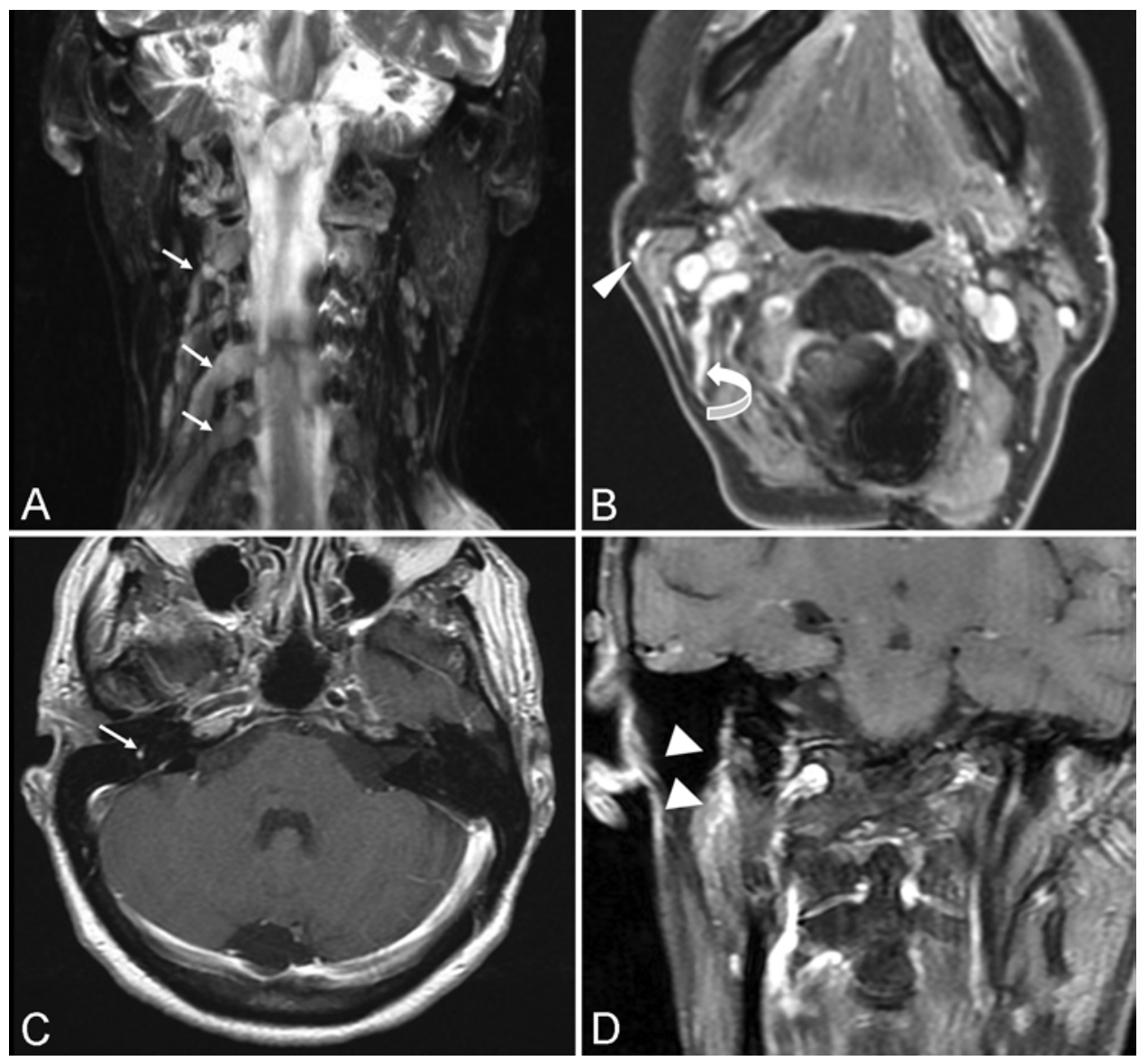

FIG. 2. A: Coronal maximum intensity projection image from a coronal T2-weighted fat-saturated sequence of the neck demonstrating nerve enlargement and T2-weighted hyperintensity greatest at C-2, C-4, and C-5 (arrows). B: Axial T1-weighted fat-saturated postcontrast image just below the right C-2 nerve, demonstrating markedly abnormal enlargement and enhancement of the nerve with extension into the great auricular (arrowhead) and lesser occipital (curved arrow) nerves. C and D: Facial nerve abnormality. Axial T1-weighted Gd-enhanced image of the brain (C) demonstrating abnormal enhancement of the mastoid segment of the right facial nerve (C, arrow). Coronal T1-weighted fat saturated Gd-enhanced image (D) demonstrating enlargement and abnormal enhancement of the parotid segment of the right facial nerve (arrowheads). 


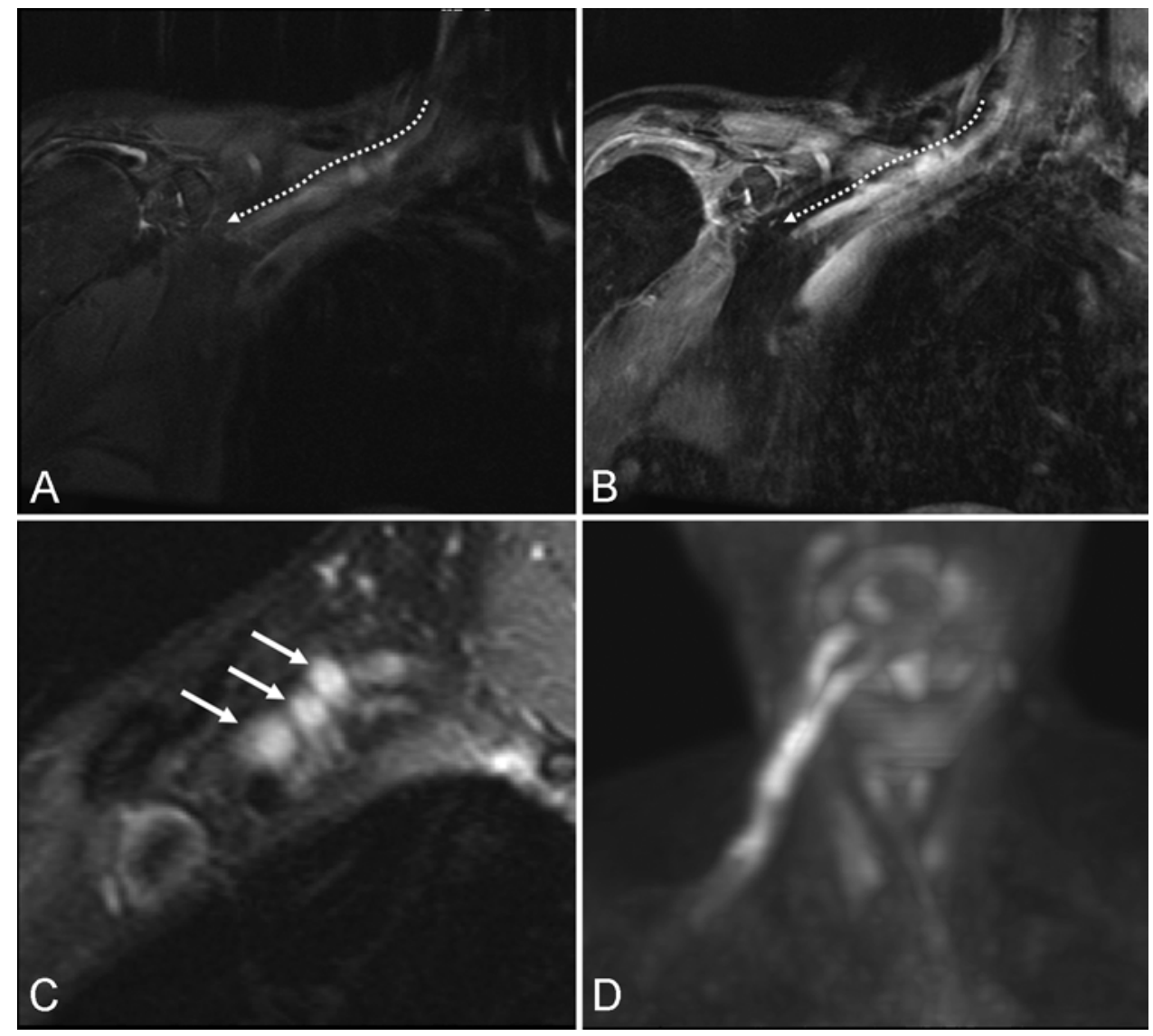

FIG. 3. A and B: Right brachial plexus coronal oblique fat-saturated T2-weighted image $(A)$ and Gd-enhanced fat-saturated image (B) demonstrating an abnormal increased T2-weighted signal and Gd enhancement extending from the C4-5 nerves into the upper trunk of the brachial plexus (dashed arrows). C: There was diffuse abnormal signal and enhancement throughout the right brachial plexus as demonstrated on the sagittal oblique fat-saturated Gd-enhanced image (arrows). D: An anterior maximum intensity projection from the FDG PET/CT study demonstrating marked abnormal FDG avidity of the right cervical nerves extending into the plexus corresponding to the abnormality on MRI, greatest at C-4 and C-5.

right facial nerve demonstrated abnormal T2-weighted hyperintensity and enhancement involving the mastoid segment of the nerve, extending inferiorly into the parotid gland (Fig. 2C and D). The spinal accessory nerve was enlarged. The trunks, divisions, and cords of the brachial plexus were enlarged as well and demonstrated marked T2-weighted hyperintensity and an infiltrative pattern of enhancement, particularly of $\mathrm{C}-5$, extending into the upper trunk (Fig. 3A-C). These findings had progressed since imaging performed 4 months earlier. FDG PET/CT scanning revealed increased linear FDG uptake along multiple cervical nerves and extending into the brachial plexus (Fig. 3D) without remote foci of increased FDG avidity to suggest metastatic disease.

\section{Operation}

An open biopsy was planned. The preoperative diagnosis was perineural spread of melanoma. The great auricular and transverse cervical nerves were targeted for biopsy rather than the brachial plexus to minimize the risks of a biopsy. This was based on the clinical involvement in those territories, the nerves' expendability, and the availability of a large amount of biopsy material, if necessary. Furthermore, these nerves were away from the radiated field and were known to communicate with the facial nerve. Biopsy sampling of the enlarged upper trunk was deferred so as to avoid further neurological deficit in the shoulder muscles, which were already severely compromised.

At operation, the previous neck scar (Fig. 4A) was reopened. The great auricular and transverse cervical nerves were enlarged and had nodular expansions and a discolored appearance (Fig. 4B). These nerves were traced proximally to the C-2 nerve and were resected (Fig. 4C). The cervical contribution to the spinal accessory nerve was preserved, although no response to stimulation was observed.

\section{Pathological Findings}

Microscopically, the architecture of the nerve was completely disrupted, with portions of the nerve being totally replaced by tumor. There were other areas of the nerve that had been partially replaced, and there were extensive associated reactive changes that also disrupted the nerve. Morphologically, in cross-section, the tumor cells were noted to have a somewhat epithelioid appearance with enlarged nuclei containing prominent nucleoli; however, in longitudinal section the tumor cells were noted to have a spindle 


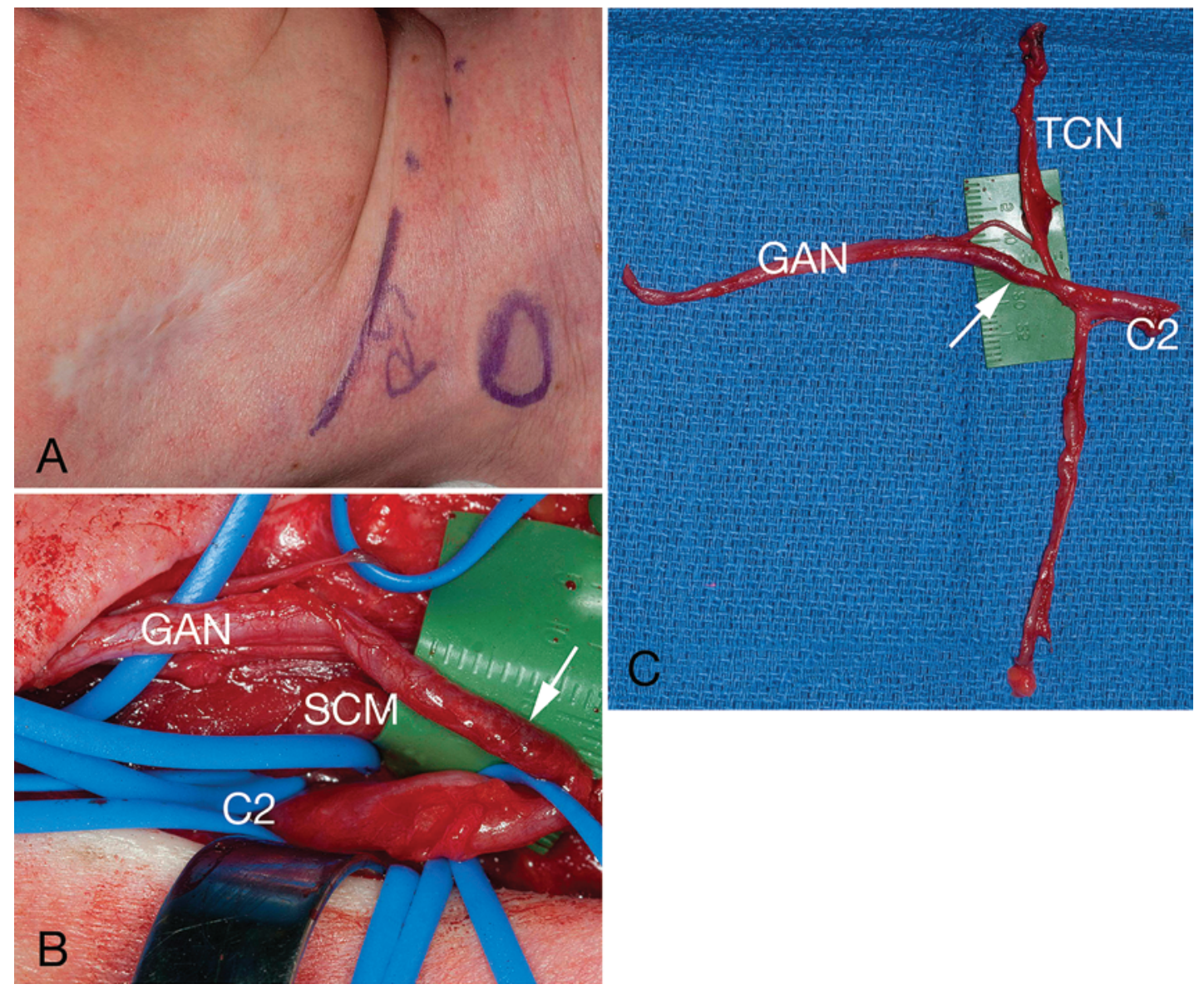

FIG. 4. Operative photographs. A: The planned incision is shown in the preexisting cervical lymphadenectomy scar. Note the proximate scar from the melanoma surgery at the mandible. The circle is on a mass within the spinal accessory nerve. B: The great auricular nerve (GAN) and transverse cervical nerve (TCN) were traced to C-2. Note the enlargement as well as focus of nodular, discolored appearance in the great auricular nerve (arrow). SCM = sternocleidomastoid muscle. C: The resected specimen.

cell morphology with elongated and atypical nuclei. The tumor was noted to be within the nerve, expanding it, and not extending out of the confines of the perineurium. The tumor was mitotically active and had an elevated level of proliferative activity indicated by Ki 67 immunohistochemical staining. On immunohistochemical analysis, the cells were strongly positive for S100 protein and had weak focal reactivity for melan-A (Fig. 5) and were negative for tyrosinase and HMB-45. Pericellular collagen type IV immunoreactivity was present and only very few axons were identified within the tumor on the neurofilament stain. Findings from C-kit and BRAF600e molecular tests were negative. Based on morphology and the immunohistochemical staining profile, a diagnosis of involvement by the patient's known desmoplastic melanoma was rendered. The tumor was reviewed in conjunction with the $\mathrm{C}-2$ nerve root biopsy findings and resection specimens that were obtained 3 years prior. Those specimens showed a spindle cell neoplasm within a somewhat myxoid background. There was some nuclear atypia. At that time, metastatic melanoma was considered in the pathological interpretation, but the diagnosis based on the available material favored neurofibroma. When these cases were reviewed retrospectively at our institution, the morphological features were compatible with a neurofibroma involving a nerve ganglion with nuclear atypia. However, when reviewed concurrently with the great auricular and transverse cervical nerve specimens, the 2011 material was interpreted as desmoplastic melanoma. Although only H \& E slides were available for review in these cases, as seen in the current resection specimens in areas that were less involved by the tumor, prominent reactive changes had a similar appearance. In the current resection specimen, the diagnosis of involvement by the patient's known desmoplastic melanoma was most evident only in portions of nerve that were almost entirely replaced by neoplasm. A cervical lymph node was found not to have tumor.

\section{Postoperative Course}

The patient was started on combined immunomodulatory therapy with ipilimumab $(3 \mathrm{mg} / \mathrm{kg}$ dose administered every 3 weeks for 4 doses) and quad-shot radiotherapy. ${ }^{6}$

\section{Discussion}

Melanoma is the least common skin cancer and represents $5 \%$ of skin cancer diagnoses; however, it accounts for $65 \%$ of skin cancer-related deaths. In the last 4 de- 

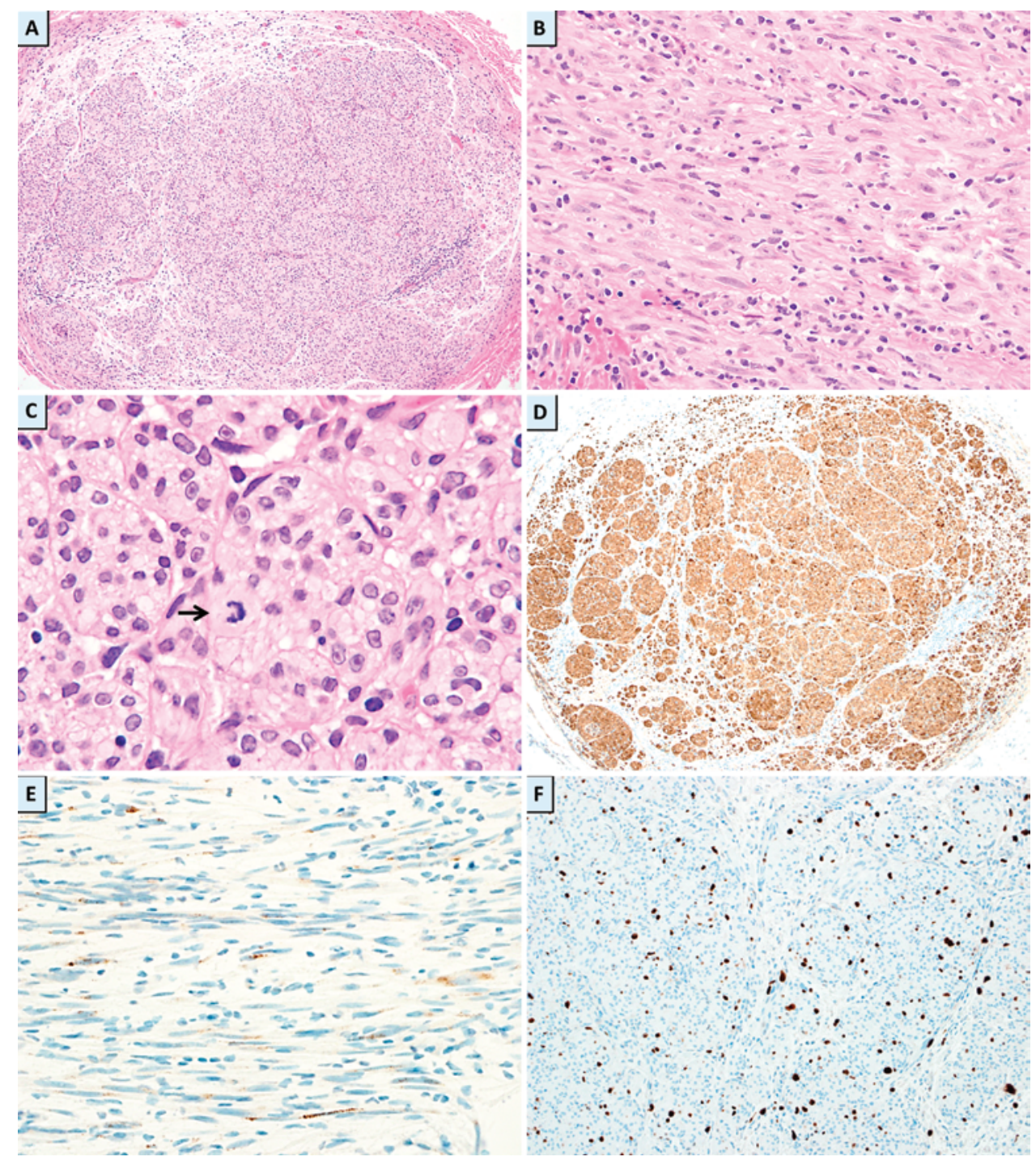

FIG. 5. Photomicrographs. A: Cross-section of nerve demonstrating near-complete replacement by the neoplasm. $H$ \& $E$, original magnification $\times 10$. B: Longitudinal section of nerve. The tumor cells have a spindle shape with elongate and atypical nuclei containing distinct nucleoli. $\mathrm{H} \& \mathrm{E}$, original magnification $\times 40$. C: Cross-section of nerve showing cells with enlarged and atypical nuclei with prominent nucleoli. A mitotic figure is also present (arrow). $\mathrm{H} \& \mathrm{E}$, original magnification $\times 60$. D: The tumor cells are strongly positive for $\mathrm{S} 100$. Original magnification $\times 10$. E: A longitudinal section shows that the tumor cells have weak reactivity for Melan-A. Original magnification $\times 40$. F: An elevated level of proliferative activity is present within the tumor. Ki 67 , original magnification $\times 20$.

cades there has been an alarming increase in its incidence, especially in industrial countries such as Australia and the United States. Overall, $20 \%$ of melanomas occur in the head and neck. ${ }^{4,7}$

The histological diagnosis of desmoplastic melanomas can be difficult. ${ }^{24}$ An important characteristic of this type of melanoma is its propensity for neural invasion, at times with characteristics termed neural transformation, which can have histological resemblance to peripheral nerve sheath tumors, including schwannoma, neurofibroma, ${ }^{20}$ and malignant peripheral nerve sheath tumor. ${ }^{2}$ This, along with the location of the main surgical specimen in the dorsal root ganglion, almost certainly accounts for the initial misdiagnosis in this case. Desmoplastic melanomas are positive for S100 and collagen type IV; however, they only rarely stain with melanocytic markers such as Melan-A, HMB-45, and tyrosinase. ${ }^{23}$ Morphologically, this tumor was not in keeping with a schwannian neoplasm. Neurofibromas would be expected to have axons present as part of the tumor. Malignant peripheral nerve sheath tumors are typically more cellular and exhibit less S100 staining.

Perineural spread has become a well-known mechanism of dissemination from different head and neck malignancies, occurring in $2.5 \%-5 \%$ of the cases and more commonly seen in carcinomas arising from the salivary glands, mucosa, and skin, as well as lymphomas and sarcomas. The most affected nerves in perineural spread are the cranial nerves, mainly the branches of the trigeminal nerve (especially V2 and V3) and the descending branch of the facial nerve; however, there are some reports of in- 
volvement of the V1 branch; cranial nerves III, VI, X, and $\mathrm{XII}$; and and the great auricular nerve as well. The spread of the tumor usually follows a retrograde (toward the central nervous system) direction, meaning that the skull base, the cranial foramina, and even intracranial structures can be affected. . $^{8,13,17,18}$ As in this case, anterograde spread can also occur. Approximately $40 \%$ of patients with perineural spread remain asymptomatic, ${ }^{18}$ making the diagnosis challenging. MRI is the most sensitive imaging modality for detecting perineural spread; however, the question of perineural spread of malignancy often arises in the setting of prior surgery and/or radiation therapy, which may complicate the MRI examination. The direct signs of perineural spread of malignancy include nerve enlargement, T2-weighted hyperintensity, and an infiltrative pattern of enhancement on Gd-enhanced images. In this case of perineural spread of melanoma to the brachial plexus, the involved nerves were markedly enlarged with a solid masslike pattern of enhancement on Gd-enhanced images. A peripheral thick or nodular pattern of enhancement is also suggestive of perineural spread of disease. The peripheral pattern of perineural disease may be challenging to separate from radiation neuritis by MRI alone. Subacutely and acutely denervated muscles demonstrate T2-weighted hyperintensity that may be an important secondary sign of perineural spread of disease..$^{10,11}$ Increased avidity with FDG PET has been described in perineural spread of melanoma, but the utility of PET/CT remains unknown. ${ }^{16}$ Currently, MRI and PET/CT are used to complement each other in the imaging evaluation of perineural spread of malignancy.

Perineural spread from melanoma in the head and neck region is well known. Chang et al. reported a series of 8 cases of melanoma affecting cranial nerves, mainly the facial and trigeminal nerves; all of the patients were symptomatic and the average time of presentation was 4.9 years after the initial diagnosis. ${ }^{4}$ Interestingly, $62 \%$ of the cases were desmoplastic, a histological subtype known to have a tendency to invade nerves; this affinity appears to be related to a high expression of p75 neurotropic receptor that is involved in the migration of the Schwann cell to the nerve. ${ }^{9,18,20}$ While regional recurrence of tumor is common because of its neurotropism, nodal metastasis is relatively uncommon, and when distant metastasis occurs, it most often involves the lung, and sometimes bone and brain..$^{10,19}$ Two cases of spinal nerve involvement have been reported. ${ }^{14,21}$ There are also several cases of melanoma involving the brachial plexus but from soft-tissue nodal compression ${ }^{7,13}$ or an unspecified mechanism ${ }^{12}$ rather than perineural spread.

This is the first known example in the literature describing a mechanism by which perineural spread of melanoma extends from a cranial nerve to the cervical and brachial plexuses. This spread has a clear anatomical basis that can be supported based on the clinical and radiological features as well as the biopsy results. At the level of the mandible, the mandibular and cervical branches of the facial nerve are known to communicate with the great auricular and transverse cervical nerves, respectively. ${ }^{22}$ We would not anticipate perineural spread from V3 to the cervical plexus based on the lack of neural communication across this axial line. ${ }^{15}$ The cervical plexus (C1-4) supplies neck muscles and various skin territories and has known communications, which can explain the spinal accessory and phrenic nerve involvement. ${ }^{1,22}$ Brachial plexus involvement could have occurred via the right phrenic nerve (C3-5) or intercommunications from C-4.11

\section{Conclusions}

Perineural spread has become a recognized mechanism for dissemination of head and neck malignancies. Most of the literature discusses perineural spread from the cranial nerves to the skull base and the central nervous system. When associated with skin malignancies, perineural spread almost exclusively occurs with squamous cell cancer. Desmoplastic melanoma commonly has neurotropic characteristics, which can account for a high rate of local recurrence when initial resection does not include a wide margin and can lead in certain circumstances to diagnostic uncertainty, including mistaken identity as nerve sheath tumors. Distant metastasis occurs in solid organs and has rarely been reported in brachial plexus or nervous system sites via perineural progression. This case report demonstrates 2 important and previously rarely recognized features, and one important diagnostic message: 1) Anterograde perineural spread of tumor from the region of the mandible to the brachial plexus can occur via communications between the cranial nerves and the cervical plexus, from the cervical plexus to the brachial plexus. 2) Desmoplastic melanoma, which commonly has neurotropic characteristics, can metastasize primarily through perineural spread. This report also demonstrates an important diagnostic message: Physicians must remain suspicious when the behavior of a lesion is not consistent with an initial diagnosis.

\section{References}

1. Banneheka S: Morphological study of the ansa cervicalis and the phrenic nerve. Anat Sci Int 83:31-44, 2008

2. Barnett SL, Wells MJ, Mickey B, Hatanpaa KJ: Perineural extension of cutaneous desmoplastic melanoma mimicking an intracranial malignant peripheral nerve sheath tumor. Case report. J Neurosurg 115:273-277, 2011

3. Breslow A: Thickness, cross-sectional areas and depth of invasion in the prognosis of cutaneous melanoma. Ann Surg 172:902-908, 1970

4. Chang PC, Fischbein NJ, McCalmont TH, Kashani-Sabet M, Zettersten EM, Liu AY, et al: Perineural spread of malignant melanoma of the head and neck: clinical and imaging features. AJNR Am J Neuroradiol 25:5-11, 2004

5. Clark WH Jr, From L, Bernardino EA, Mihm MC: The histogenesis and biologic behavior of primary human malignant melanomas of the skin. Cancer Res 29:705-727, 1969

6. Corry J, Peters LJ, Costa ID, Milner AD, Fawns H, Rischin D, et al: The 'QUAD SHOT'-a phase II study of palliative radiotherapy for incurable head and neck cancer. Radiother Oncol 77:137-142, 2005

7. Garbe C, Leiter U: Melanoma epidemiology and trends. Clin Dermatol 27:3-9, 2009

8. Ginsberg LE, Eicher SA: Great auricular nerve: anatomy and imaging in a case of perineural tumor spread. AJNR Am J Neuroradiol 21:568-571, 2000

9. Iwamoto S, Odland PB, Piepkorn M, Bothwell M: Evidence 
that the $\mathrm{p} 75$ neurotrophin receptor mediates perineural spread of desmoplastic melanoma. J Am Acad Dermatol 35:725-731, 1996

10. Jaroszewski DE, Pockaj BA, DiCaudo DJ, Bite U: The clinical behavior of desmoplastic melanoma. Am J Surg 182:590-595, 2001

11. Johnson EO, Vekris M, Demesticha T, Soucacos PN: Neuroanatomy of the brachial plexus: normal and variant anatomy of its formation. Surg Radiol Anat 32:291-297, 2010

12. Kori SH, Foley KM, Posner JB: Brachial plexus lesions in patients with cancer: 100 cases. Neurology 31:45-50, 1981

13. Kozić D, Njagulj V, Gaćeša JP, Semnic R, Prvulović N: Perineural tumor spread-interconnection between spinal and cranial nerves. J Neurol Sci 323:254-256, 2012

14. Kwon SC, Rhim SC, Lee DH, Roh SW, Kang SK: Primary malignant melanoma of the cervical spinal nerve root. Yonsei Med J 45:345-348, 2004

15. Ladak A, Tubbs RS, Spinner RJ: Mapping sensory nerve communications between peripheral nerve territories. Clin Anat 27:681-690, 2014

16. Lee TJ, Glastonbury CM, Buckley AF, Eisele DW, Hawkins RA: Perineural spread of melanoma demonstrated by F-18 FDG PET With MRI and pathologic correlation. Clin Nucl Med 33:106-108, 2008

17. Maroldi R, Farina D, Borghesi A, Marconi A, Gatti E: Perineural tumor spread. Neuroimaging Clin N Am 18:413-429, xi, 2008

18. Moonis G, Cunnane MB, Emerick K, Curtin H: Patterns of perineural tumor spread in head and neck cancer. Magn Reson Imaging Clin N Am 20:435-446, 2012

19. Murali R, Zannino D, Synnott M, McCarthy SW, Thompson JF, Scolyer RA: Clinical and pathological features of metas- tases of primary cutaneous desmoplastic melanoma. Histopathology 58:886-895, 2011

20. Newlin HE, Morris CG, Amdur RJ, Mendenhall WM: Neurotropic melanoma of the head and neck with clinical perineural invasion. Am J Clin Oncol 28:399-402, 2005

21. Skarli SO, Wolf AL, Kristt DA, Numaguchi Y: Melanoma arising in a cervical spinal nerve root: report of a case with a benign course and malignant features. Neurosurgery 34:533-537, 1994

22. Standring S: Gray's Anatomy: The Anatomical Basis of Clinical Practice, ed 40. London: Elsevier Churchill Livingstone, 2005

23. Weissinger SE, Keil P, Silvers DN, Klaus BM, Möller P, Horst BA, et al: A diagnostic algorithm to distinguish desmoplastic from spindle cell melanoma. Mod Pathol 27:524-534, 2014

24. Wood BA: Desmoplastic melanoma: recent advances and persisting challenges. Pathology 45:453-463, 2013

\section{Author Contributions}

Conception and design: Spinner, Restrepo. Acquisition of data: Spinner, Howe, Lachance. Analysis and interpretation of data: Jentoft. Drafting the article: Spinner, Restrepo, Howe. Critically revising the article: Spinner, Restrepo, Markovic, Lachance. Reviewed submitted version of manuscript: Spinner, Howe, Jentoft, Markovic, Lachance. Approved the final version of the manuscript on behalf of all authors: Spinner.

\section{Correspondence}

Robert J. Spinner, Mayo Clinic, 200 First St. SW, Gonda 8-214, Rochester, MN 55905. email: spinner.robert@mayo.edu. 